\title{
Optical Rotatory and Circular Dichroic Scattering
}

\author{
Werner Kaminsky, ${ }^{*}$ Morten Andreas Geday, ${ }^{\dagger}$ Javier Herreros-Cedrés, ${ }^{\ddagger}$ and Bart Kahr* \\ Department of Chemistry, Box 351700, University of Washington, Seattle, Washington 98195-1700
}

Received: June 23, 2002; In Final Form: November 5, 2002

\begin{abstract}
Optical effects are observed in regularly dyed crystals that serve to mimic optical rotation and circular dichroism by rotating the azimuth and increasing the ellipticity of linearly polarized light traversing the samples. However, these effects to which we give the names optical rotatory scattering and circular dichroic scattering do not transform upon rotation of the sample like intrinsic optical rotation and intrinsic circular dichroism. We ascribe this behavior to new scattering consequences that arise here in crystals of $\mathrm{K}_{2} \mathrm{SO}_{4}$ containing oriented azo dyes that have been arranged and overgrown in particular growth sectors. Requisite for the apparent optical rotation and circular dichroism is a bias in the inclination of the induced dipoles with respect to the principal light propagation modes of the medium. This unique situation is a consequence of the anisotropy of growth that is unlikely to occur in other dye-doped systems such as polymers or liquid crystals.
\end{abstract}

\section{Introduction}

We set out to apply newly developed methods for the measurement of optical rotation in anisotropic media ${ }^{1}$ to evaluate the fine structure of mixed crystals. Specifically, we aimed to image optical rotation (OR) in simple centrosymmetric crystals that had adsorbed, oriented, and overgrown chiral dye molecules. Chiroptical effects such as OR and circular dichroism (CD) were expected when equilibrium racemic mixtures of dyes selectively recognized chiral facets of achiral crystallographic hosts.

Chiral discrimination of organic compounds by minerals has been a rich subject for speculation since the early suggestions by Goldschmidt ${ }^{2}$ and Bernal $^{3}$ that chiral clays or quartz may have been responsible for the origin of the asymmetry in biopolymers. ${ }^{4}$ Scientists at the Weizmann Institute ${ }^{5}$ and others ${ }^{6}$ have subsequently worked out many mechanisms of the chiral discrimination of molecules by crystal surfaces. These studies have involved the interactions of racemic mixtures of enantiomers of fixed configurations with homochiral crystals ${ }^{7}$ (Figure 1a) or resolved chiral compounds of fixed configurations with mirror image crystal facets (Figure 1b).

We have systematically studied the process of dyeing crystals. ${ }^{8}$ In many cases, chiral dyes were adsorbed by chiral crystal surfaces. Working out association mechanisms required that we first establish whether the adsorptions were indeed enantioselective and also determine the absolute configurations of the chiral faces and their respective adsorbates. The examples illustrated herein were inspired by Buckley, who described in 1934 a mixed crystal of $\mathrm{K}_{2} \mathrm{SO}_{4}$ that had been grown in the presence of the dye brilliant Congo $\mathrm{R}^{9}\left(\mathbf{1}\right.$, Colour Index ${ }^{10}$ no. 23570 , Figure 2). The dye selectively recognized the $\{111\}$ facets of $\mathrm{K}_{2} \mathrm{SO}_{4}$ with $m m m\left(D_{2 h}\right)$ symmetry. ${ }^{11}$ Any face with a 0 index is parallel to a mirror plane and achiral. Thus, the eight $\{111\}$ faces are chiral and pairwise enantiomorphously related

* To whom correspondence should be addressed. E-mail: kaminsky@ wintensor.com,kahr@chem.washington.edu.

$\dagger$ Present address: Clarendon Laboratory, Department of Physics, University of Oxford, Oxford OX1 3PU, United Kingdom.

$\doteqdot$ Present address: Departamento de Física Básica, Facultad de Física, Universidad de La Laguna, La Laguna 38206, Tenerife Spain.

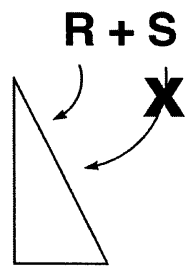

a

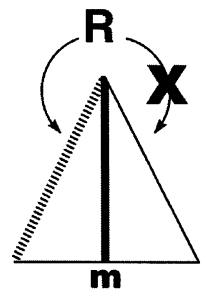

b

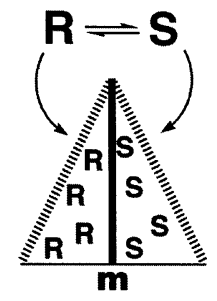

C
Figure 1. Schematic illustration of three examples of chiral discrimination of molecules by adsorption to crystals: (a) Resolution of a racemate with fixed configurations by a chiral crystal. A typical example would be the selective adsorption of racemic amino acids by quartz. ${ }^{7}$ (b) Assignment of the absolute configuration of enantiomorphous faces using a resolved chiral molecule. Here, mirror symmetric faces of glycine, for example, are disturbed differently by a resolved chiral molecule of fixed configuration such as D-alanine. ${ }^{5}$ (c) Buckley's system, $\mathrm{K}_{2} \mathrm{SO}_{4} / \mathbf{1}$, is a convolution of parts a and $\mathrm{b}$ where both enantiomers and enantiomorphous faces are present, a scenario further complicated by the fact that the enantiomers are in rapid equilibrium. ${ }^{9}$ Here, enantioselectivity has not been observed but only presumed.

to one another by reflections through the three orthogonal mirror planes (Figure 3). Consistent with this symmetry, the mixed crystals developed patterns of color that resembled a Maltese cross. Brilliant Congo $\mathrm{R}$ is a biaryl dye that is chiral in its ground state although it exists as an equilibrium racemic mixture of rapidly interconverting enantiomers in solution. Therefore, it is likely that the chiral surfaces adsorbed the enantiomers selectively. We set out to determine whether the extent of the enantioselection would lend itself to measurement.

To demonstrate enantioselectivity, we needed to determine whether the included dyes showed a chiroptical property such as $\mathrm{OR}$ or $\mathrm{CD}$ inside the crystal because dissolution would instantly restore the solution equilibrium racemic mixture. This plan was complicated by the longstanding difficulty of measuring chiroptical properties in anisotropic materials that exhibit much larger linear birefringence (LB) and linear dichroism (LD). $\mathrm{K}_{2} \mathrm{SO}_{4} / \mathbf{1}$ (throughout, this notation indicates a $\mathrm{K}_{2} \mathrm{SO}_{4}$ crystal containing small quantities of the numbered dye) mixed crystals were further complicated by the need to measure a 

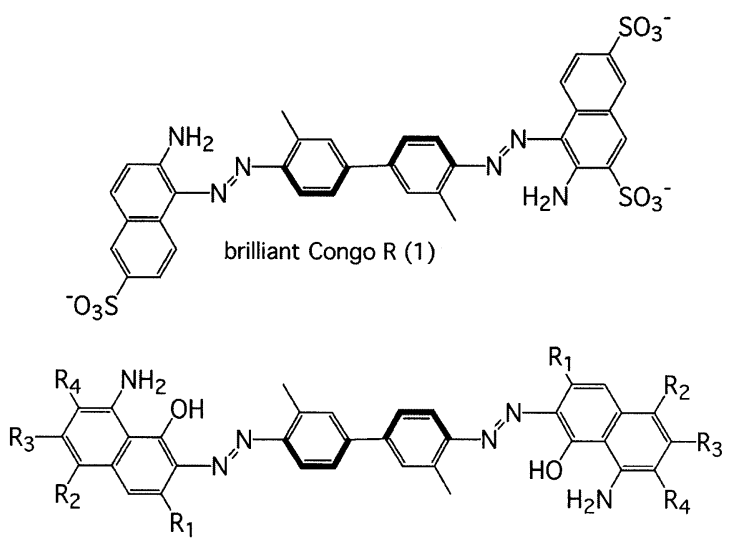

Evan's blue (2): $\mathrm{R}_{1}, \mathrm{R}_{3}=\mathrm{H} ; \mathrm{R}_{2}, \mathrm{R}_{4}=\mathrm{SO}_{3}{ }^{-}$

trypan blue (3): $\mathrm{R}_{1}, \mathrm{R}_{3}=\mathrm{SO}_{3} ; \mathrm{R}_{2}, \mathrm{R}_{4}=\mathrm{H}$

Figure 2. Biaryl azo dyes.

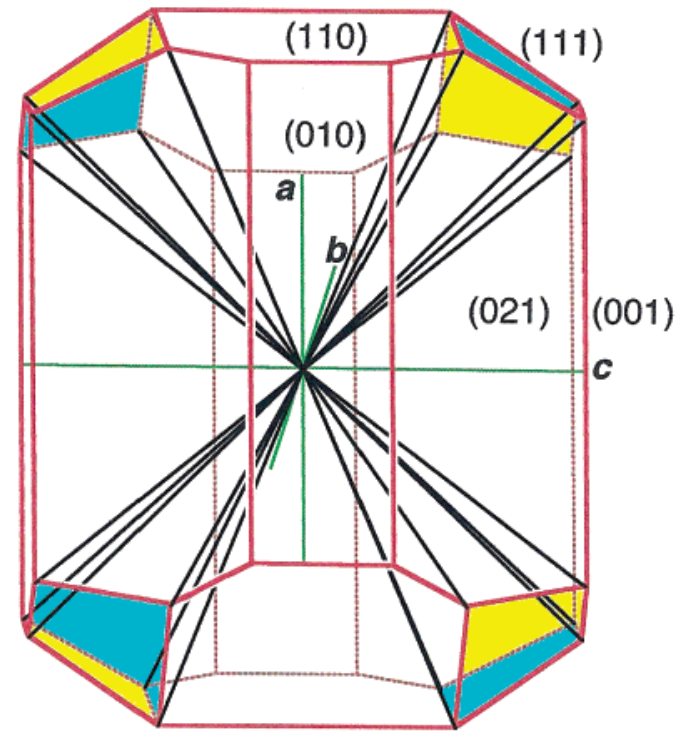

Figure 3. Idealized representation of the $\mathrm{K}_{2} \mathrm{SO}_{4}$ habit (red). Black lines delineate the $\{111\}$ growth sectors whose enantiomorphous faces are distinguished as blue and yellow, respectively. Green lines are the crystallographic axes that also define the principal directions of the optical indicatrix.

chiroptical response of a minor crystal component; from previous experience, we surmised that there was one mole of dye per $\sim 10^{4}$ moles of salt in one of the colored regions of Buckley's crystal. ${ }^{15}$

We attempted to grow Buckley's $\mathrm{K}_{2} \mathrm{SO}_{4} / \mathbf{1}$. Unable to find a commercial supplier of $\mathbf{1}$, we synthesized it using standard techniques involving biaryl diazotization and electrophilic aromatic substitution of the corresponding naphthylamines. ${ }^{12}$ Unfortunately, our sample of $\mathbf{1}$ failed to dye the $\{111\}$ growth sectors. This was not a large concern because the structures of many of the dyes described by Buckley in the 1930s were misassigned. ${ }^{13}$ We nevertheless succeeded in preparing Buckleylike Maltese crosses in $\mathrm{K}_{2} \mathrm{SO}_{4}$ with the commercially available dyes Evan's blue (2; CI no. 23860) and trypan blue (3; CI no. 23850), which as sulfonated biarylazo dyes share many of the structural characteristics of brilliant Congo R.

$\mathrm{K}_{2} \mathrm{SO}_{4} / 2$ or 3 crystals were remarkable because between crossed polarizers a slight rotation of the thin plates from extinction made the purple $\{111\}$ sectors appear alternately red and green. We provisionally ascribed this to optical rotatory dispersion. For slight rotations away from the extinction position, part of the spectrum would remain extinct if the OR were strongly wavelength-dependent. Indeed, we detected polarimetric signals associated with the oriented dyes that were consistent with OR and CD (see section 2.2) and seemed to confirm our qualitative observations, but surprisingly, they did not transform like $\mathrm{OR}$ and $\mathrm{CD}$. Although symmetry requires oppositely signed responses from oscillators adsorbed through mirror image crystallographic facets (Figure 4), thereby suggesting an enantioselective growth process, the signals also changed sign with the rotation of the sample through $90^{\circ}$ about the wave vector of the incident light or by reversing the direction of the wave vector. This behavior was inconsistent with intrinsic OR and CD. Here, we interpret our observations in terms of optical effects that have not heretofore been described and to which we give the names optical rotatory scattering (ORS) and circular dichroic scattering (CDS). ${ }^{14}$ Presumably, the effects are new because of the special characteristics of dyed crystals not matched in other materials.

\section{Experimental Section}

2.1. Sample Preparation. $\mathrm{K}_{2} \mathrm{SO}_{4}$ (Baker) crystals containing $\mathbf{2}$ and $\mathbf{3}$ (Aldrich) were grown by evaporating aqueous solutions at room temperature. A $250-\mathrm{mL}$ beaker containing $100 \mathrm{~mL}$ of distilled water was charged with $17.6 \mathrm{~g}$ of $\mathrm{K}_{2} \mathrm{SO}_{4}$ and $5 \mathrm{~mL}$ of a $1 \mathrm{mg} / \mathrm{mL}$ solution of either $\mathbf{2}$ or $\mathbf{3}$. The solution was heated until all of the $\mathrm{K}_{2} \mathrm{SO}_{4}$ was dissolved and then filtered. The filtrate was returned to a crystallizing dish, covered with a watch glass, and cooled to room temperature overnight, during which time there was considerable precipitation of $\mathrm{K}_{2} \mathrm{SO}_{4}$ microcrystals and colloidal dye aggregates. The precipitates were removed by gravity filtration. The resulting solution was allowed to stand at room temperature for another day. Additional filtrations of ill-formed precipitates were sometimes required. Finally, after approximately 2 days, large $\left(\sim 5 \times 5 \times 2 \mathrm{~mm}^{3}\right) \mathrm{K}_{2} \mathrm{SO}_{4}$ crystals containing purple Maltese crosses composed of dyed $\{111\}$ sectors were obtained. Sometimes the $\{110\}$ and $\{010\}$ sectors were faintly colored.

Crystals were cut, shaped, and polished with a string saw, sandpaper, and alumina grit, respectively. Typically, the $\{111\}$ sectors contained $10^{-4}$ moles of $2\left(\epsilon_{\text {soln }}=1.8 \times 10^{4} \mathrm{~L} \mathrm{M}^{-1}\right.$ $\left.\mathrm{cm}^{-1}\right)$ and $\mathbf{3}\left(\epsilon_{\mathrm{soln}}=6.9 \times 10^{4} \mathrm{~L} \mathrm{M}^{-1} \mathrm{~cm}^{-1}\right)$ per mole of $\mathrm{K}_{2} \mathrm{SO}_{4}$. We previously measured the dichroic ratios using a polarizing microscope-based spectrophotometer. ${ }^{15}$ In the (010) plane, the dipoles of 2 and 3 were inclined by $54 \pm 2^{\circ}$ and $57 \pm 2^{\circ}$ with respect to the $a$ axis, respectively.

2.2. Optical Characterization. To measure OR (circular birefringence, $\Delta n_{\mathrm{CB}}=n_{\mathrm{R}}-n_{\mathrm{L}}$ ) along a general direction of a crystal, one has to deconvolve the influence of $\mathrm{LB}\left(\Delta n_{\mathrm{LB}}=n^{\prime \prime}\right.$ $-n^{\prime}$ ) and linear dichroism (LD) ${ }^{16,17}$ (In practice, our samples are so thin that LD is not significant.) The intensity of light passing through a polarizer, chiral anisotropic sample, and analyzer contains the necessary information, in principle, for extracting OR. However, the implementation of this idea prior to the invention of electrophotometry and stable, high-intensity light sources was impossible until relatively recently. For generations, most researchers conceded that measuring OR in the presence of large LB was not a realistic goal.

In 1983, Uesu and Kobayashi pushed through this impasse by using lasers, photon counting, and computerized modulation of polarizer and analyzer orientations to determine OR in crystals for directions off the optic axes. ${ }^{18}$ They called this experiment HAUP (high-accuracy universal polarimetry), whose basic geometry is given in Figure 5. 


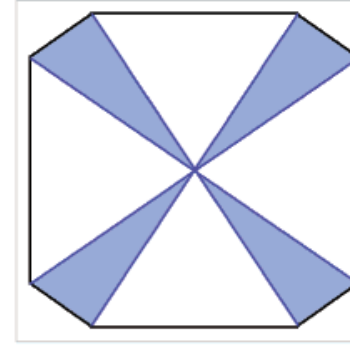

a)

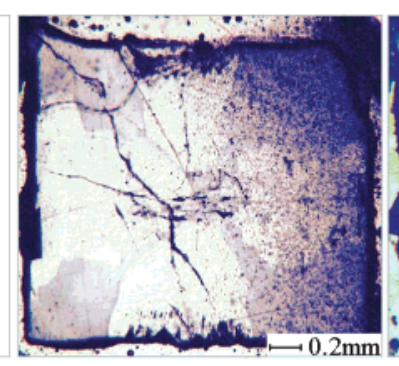

b)

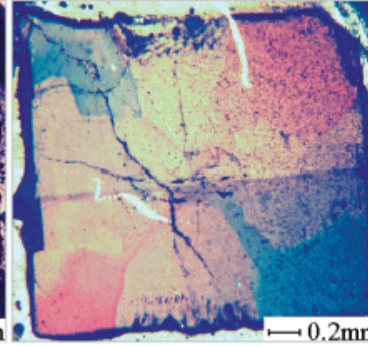

c)

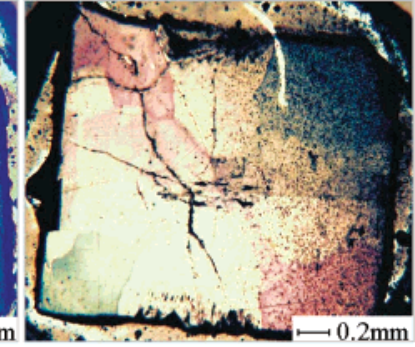

d)

Figure 4. (a) Idealized representation of a (010) slice of $\mathrm{K}_{2} \mathrm{SO}_{4} / 3$, where the purple dye is indicated in the $\{111\}$ sectors in cross section. (b) A 90- $\mu$ m-thick (010) plate $\left(\sim 2 \times 2 \mathrm{~mm}^{2}\right)$ of $\mathrm{K}_{2} \mathrm{SO}_{4} / 3$ prepared from a crystal as in Figure 3. (This crystal is not 4-fold-symmetric. The slice merely came from crystals whose morphology was inhibited on [100], thus giving a square cross section.) (c, d) Between crossed polarizers with the sample rotated a few degrees counterclockwise and clockwise, respectively, away from the extinction position. Mirror planes divide the plate into four enantiomorphous $\{111\}$ growth sectors. Symmetry arguments require that a chiroptical effect such as OR would show opposite signs in adjacent sectors. Alternating colors would be expected if the effect was highly dispersive.

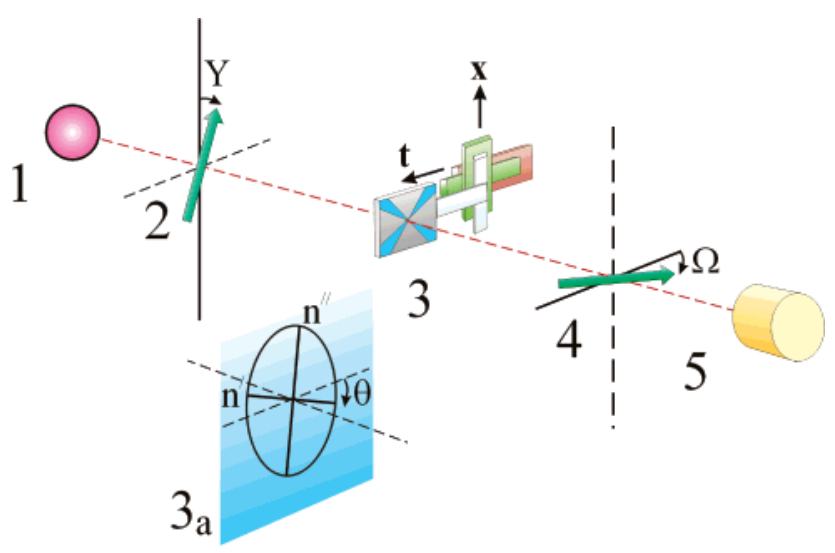

Figure 5. HAUP optical train. (1) light source, (2) polarizer, rotation angle $Y$; (3) sample and translation stage with translation directions $t$ and $x$; (3a) $\theta$ : extinction angle of the birefringent cross section with refractive indices $n^{\prime}$ and $n^{\prime \prime} ;(4)$ analyzer, rotation angle $\Omega$; (5) photodiode.

According to the Jones matrix formalism, ${ }^{19}$ a sample that shows $\mathrm{LB}\left(\delta=\left(2 \pi \Delta n_{\mathrm{LB}} L\right) / \lambda\right)$ and $\mathrm{OR}(\varphi)$ as well as $\mathrm{CD}(\eta)$ is given by the following:

$$
\begin{aligned}
& M(\delta, \varphi, \eta)= \\
& \left.\qquad \begin{array}{ll}
\mathrm{e}^{\mathrm{i} \delta / 2} & -2(\varphi+\mathrm{i} \eta)(\sin (\delta / 2)) / \delta \\
2(\varphi+\mathrm{i} \eta)(\sin (\delta / 2)) / \delta & \mathrm{e}^{(-\mathrm{i} \delta) / 2}
\end{array}\right]
\end{aligned}
$$

In a HAUP experiment, the sample at extinction angle $\theta_{0}$ is placed between two orthogonal polarizers that are rotated about small angles $Y$ and $\Omega$ (Figure 5). The optical train is represented by a string of matrices where $A$ is the light amplitude with rotation matrices for the polarizer $\left(R_{Y}\right)$, analyzer $\left(R_{\Omega}\right)$, and sample $\left(R_{\theta_{0}}\right)$ :

$$
A=R_{\Omega}^{T}\left[\begin{array}{ll}
0 & 0 \\
0 & 1
\end{array}\right] R_{\Omega} R_{\theta_{0}}^{T} M R_{\theta_{0}} R_{Y}\left[\begin{array}{l}
1 \\
0
\end{array}\right]
$$

with

$$
\begin{array}{r}
R_{\Omega}=\left[\begin{array}{ll}
\cos \Omega & -\sin \Omega \\
\sin \Omega & \cos \Omega
\end{array}\right] \quad R_{\mathrm{Y}}=\left[\begin{array}{ll}
\cos Y & -\sin Y \\
\sin Y & \cos Y
\end{array}\right] \\
R_{\theta_{0}}=\left[\begin{array}{ll}
\cos \theta_{0} & -\sin \theta_{0} \\
\sin \theta_{0} & \cos \theta_{0}
\end{array}\right]
\end{array}
$$

The result of these operations is written approximately as a biquadratic polynomial that is normalized to the amplitudes of $Y^{2}$ and $\Omega^{2}$ :

$$
\frac{I}{I_{0}}=A^{*} \cdot A=a_{0}+a_{1} \Omega+a_{2} Y+a_{3} \Omega Y+\Omega^{2}+Y^{2}
$$

with

$$
\begin{aligned}
& \frac{1}{2} a_{1}=-\theta(\delta)+\varphi(\delta)=\left(\theta_{0}-\frac{\eta}{\delta}\right)(\cos \delta-1)+\frac{\varphi_{0}}{\delta} \sin \delta \\
& \frac{1}{2} a_{2}=\theta(\delta)+\varphi(\delta)=\left(\theta_{0}-\frac{\eta}{\delta}\right)(1-\cos \delta)+\frac{\varphi_{0}}{\delta} \sin \delta \\
& \frac{1}{2} a_{3}=\cos \delta
\end{aligned}
$$

The first term $\left(a_{0}\right)$ is the overall offset in the intensity measurement. Parameters $\varphi$ and $\theta$ are found from combinations of the parameters $a_{i}: \varphi=1 / 4\left(a_{1}+a_{2}\right) ; \theta\left(\theta_{0}, \eta\right)=1 / 4\left(a_{2}-a_{1}\right)$. Parasitic ellipticities of the polarizer and analyzer were corrected by calibrating the final results to nonoptically active crystals (i.e., nondyed crystal regions in centrosymmetric $\mathrm{K}_{2} \mathrm{SO}_{4}{ }^{18}$ ).

HAUP applies reliably to smaller values of $\mathrm{LB}$; the contribution to the intensity from OR varies as does $\sin (\delta) / \delta .{ }^{20}$ Because $\mathrm{K}_{2} \mathrm{SO}_{4}$ birefringence $\left(\Delta n=n_{\mathrm{c}}-n_{\mathrm{a}}\right)$ along [010] is small, $0.0026,\left(n_{\mathrm{a}}=1.4928, n_{\mathrm{b}}=1.4916, n_{\mathrm{c}}=1.4954\right)^{21}$ and crystals were polished to less than $100 \mu \mathrm{m}$, HAUP was, in principle, appropriate for the measurement of OR and was employed with a polarimeter operating at $670 \mathrm{~nm}$, which is described elsewhere. ${ }^{22}$ However, because we are dealing with heterogeneous crystals, the polarimeter was fitted with a translation stage in order to produce topographs of optical parameters. In this way, we made maps comprising $100 \times 100$ pixels at a resolution of about $30 \mu \mathrm{m}$ per pixel. ${ }^{23}$ In the Fourier treatment of the intensity data, the apparent extinction angle convolved with circular dichroism $\left(\theta\left(\theta_{0}, \eta\right)\right)$, the phase factor $(\delta)$, and the apparent OR $(\varphi)$ were unfolded. ${ }^{22}$

Topographs of a $\mathrm{K}_{2} \mathrm{SO}_{4} / 3$ (010) section are shown in Figure 6 , where the dyed regions exhibit signals consistent with our expectations for linearly birefringent, optically rotatory, and circularly dichroic samples (cf. Figure 4). The first row in Figure 6 represents $\delta$. The sign of $\delta$ changes as the fast and slow axes are exchanged. In the middle row, the sign of $\varphi_{0}$ changes in the top left quadrant with sample reorientation. The third row of images in Figure 6 shows the apparent extinction $\theta_{0}-\eta / \delta$. From the sign of $\theta$ observed in the dyed regions, we can derive the sign of $\eta$. 


\begin{tabular}{|c|c|c|c|c|}
\hline $670 \mathrm{~nm}$ & reference & $90^{\circ}$ rotation & $90^{\circ}$ rotat./horiz. flip & horizontal flip \\
\hline $\begin{array}{c}\mathbb{E}^{3} \\
-2 \\
1 \\
-1 \\
0 \\
-1 \\
-2 \\
-3 \\
\frac{\delta}{0.4 \mathrm{~mm}}\end{array}$ & & th & & 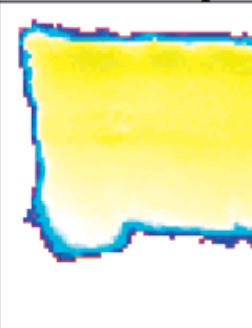 \\
\hline $\operatorname{sign} \delta$ & + & - & - & + \\
\hline $\begin{array}{c}\int_{-1.5}^{1.5} \\
\Phi_{-1.5} \\
\frac{\varphi_{0}}{0.4 \mathrm{~mm}}\end{array}$ & $y^{x}$ & 8 & 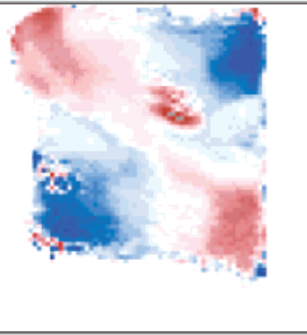 & 8 \\
\hline $\operatorname{sign} \varphi, \eta$ & + & - & + & - \\
\hline 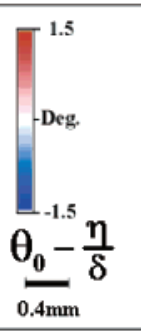 & 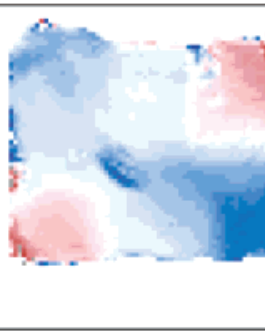 & 5 & 4 & tis \\
\hline $\operatorname{sign} \theta$ & $(+\delta) \cdot(+\eta)=+$ & $(-\delta) \cdot(-\eta)=+$ & $(-\delta) \cdot(+\eta)=-$ & $(+\delta) \cdot(-\eta)=-$ \\
\hline
\end{tabular}

Figure 6. Optical topographs of the $\mathrm{K}_{2} \mathrm{SO}_{4} / 3(010)$ plate $(\sim 90 \mu \mathrm{m})$ from Figure 4 using the scanning HAUP technique. Top row: phase factor $\delta$ $(=2 \pi \Delta n L / \lambda), \lambda=670 \mathrm{~nm}$ for various crystal orientations. The shape of the sample seen in the images in this row serves as a guide for the eye as the sample is reoriented in successive columns. Middle row: apparent optical rotation $\varphi_{0}$. Bottom row: apparent extinction $\theta$, which is a function of extinction $\left(\theta_{0}\right)$ and of circular dichroism $(\eta)$. The signs of the quantities are referred to in the top left quadrant in the first image. "Rotation" $=90^{\circ}$ rotation about wave vector. "Flip" $=180^{\circ}$ rotation about the horizontal or vertical axes of the crystal plate.

Because $\varphi$ is modulated by phase $\delta$ as

$$
\varphi(\delta)=\varphi_{0} \frac{\sin \delta}{\delta}
$$

the sign of the signal was independent of $\delta$. However, upon reorientation of the sample plate, $\varphi$ did not behave as expected; its sign changed whenever the sample was turned by $90^{\circ}$ about the wave vector ("rotation") or rotated $180^{\circ}$ around the vertical or horizontal axes perpendicular to the wave vector ("flip"). Intrinsic OR (and CD) would be invariant to these transformations.

However, a $90^{\circ}$ rotation did not change $\theta$ as seen in the bottom row of Figure 6 . Because $\delta$ changed sign with a $90^{\circ}$ rotation, we conclude that contributions $\eta$ must have changed sign as well:

$$
\theta(\delta)=\left(\theta_{0}-\frac{\eta}{\delta}\right)(1-\cos \delta)
$$

When the sample was flipped, $\theta$ did change sign. This again indicated a sign change of $\eta$ since phase $\delta$ is invariant under a flip.

Since $\delta$ is not much larger than $\pi$, the $\varphi$ rotation was seen with an ordinary polarizing microscope (Figure 4 ). When the sample was slightly rotated off of the extinction position, it gave a strong dispersion of the rotatory effect. Part of the spectrum was extinct for small angular rotations $\left(2-5^{\circ}\right)$. This is visible in the colors transmitted by the dyed sectors (Figure 4). To compensate for the effect of a positive rotation at $670 \mathrm{~nm}$, we had to tilt the sample counterclockwise or rotate the analyzer clockwise. The apparent color of the sample lacked the red component of the spectrum, and the quadrant having a positive $\varphi$ in the first image in Figure 6 appeared green. The orange color seen inside the dyed region of an adjacent quadrant would suggest that the spectrum lacked the blue component and that the rotation of that sector was levorotatory.

We can now see that the chromatic effects in Figure 4 can be explained in terms of a signal that resembles OR and excludes the possibility of a rotation of the optical indicatrix. We can then infer that the third row of Figure 6 represents a signal related to $\mathrm{CD}$ and is also not due to indicatrix rotation.

We also studied the effect of $\mathbf{2}$ on the optical properties of $\mathrm{K}_{2} \mathrm{SO}_{4}$ (Figure 7). The crystal section $\mathrm{K}_{2} \mathrm{SO}_{4} / 2$ that we examined was appealing because the dye was also included through the achiral (010) face, which can be seen in Figure 7a as a central rectangle linking the $\{111\}$ "arms" of the Maltese cross (one of which, the lower left, is barely visible, presumably because the corresponding $\{111\}$ growth sector was poorly developed) and therefore acted as an internal standard. We again saw signals that mimicked OR and CD in the $\{111\}$ sectors but not in the (010) sector. Thus, as anticipated and required by symmetry, it would appear that we had observed $\varphi$ and $\theta$ only when 2 interacted with a chiral face. However, we observed the same dependence on the sign of $\varphi$ and $\theta$ with rotations and flips as 
a)

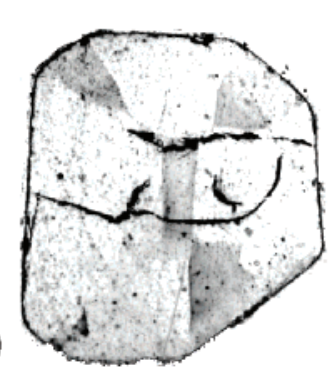

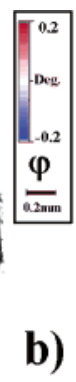

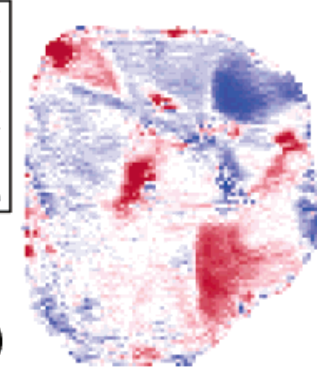

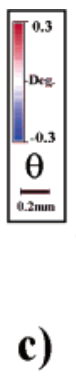

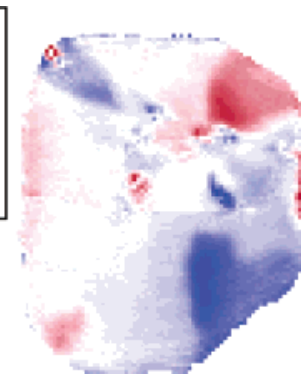

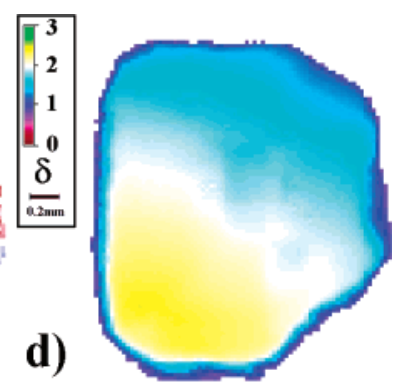

Figure 7. Section of a Maltese cross $\mathrm{K}_{2} \mathrm{SO}_{4} / 2$ (010) crystal (ca. $\left.100 \mu \mathrm{m}\right)$. The dye was oriented and overgrown by the $\{010\}$ and $\{111\}$ growth sectors. (a) Photograph. Triangular colored regions from $\{111\}$; central rectangle from $\{010\}$. (b) Optical rotation. (c) Apparent extinction. (d) Phase factor $\delta$, wavelength $670 \mathrm{~nm}$. The graded retardation is a mere consequence of the imposition of a wedge shape to the sample during hand polishing.

for $\mathrm{K}_{2} \mathrm{SO}_{4} / \mathbf{3}$. To make sense of the unexpected transformation properties of $\mathrm{K}_{2} \mathrm{SO}_{4} / 3$ and $\mathrm{K}_{2} \mathrm{SO}_{4} / 2$, we propose two new optical effects: optical rotatory scattering (ORS) and circular dichroic scattering (CDS). ORS and CDS, consequences of biased Rayleigh scattering (see below), mimic in a single measurement intrinsic $\mathrm{OR}$ and $\mathrm{CD}$ by effecting an azimuthal rotation and ellipticity in linearly polarized light traversing the sample.

\section{Discussion}

3.1. Qualitative Model for Optical Rotatory Scattering. Light scattering depends on the size of the scattering object relative to the wavelength of the incident light and the relative disposition of the scatterers with respect to one another. ${ }^{24}$ Moreover, Verreault had recognized that nonisotropic scattering centers can affect the state of light polarization. ${ }^{25}$ Dye molecules $\mathbf{2}$ and $\mathbf{3}$ ( $\sim 2 \mathrm{~nm}$ long) are much smaller than the wavelength of visible light $(300-700 \mathrm{~nm})$. At a concentration of about $10^{-4}$, the distance between dye molecules is ca. 10-100 nm. Such dilute solutions will not always contain pairs of molecules whose scattered waves interfere destructively (Figure 8).

Dyes 2 and $\mathbf{3}$ will have strong light-induced dipoles, ${ }^{26,27} \mathbf{p}$. The induced dipole $\mathbf{p}$ is related to the field $\mathbf{E}$ of the incident light wave through the polarization tensor $\alpha_{i j}$ :

$$
\mathbf{p}_{i}=\alpha_{i j} \mathbf{E}_{j}
$$

If we assume strongly preferred transition dipole moments, the polarization tensor is represented in the following way in the reference system of the molecule. (We simplify the tensor by assuming that this direction is in a plane perpendicular to the wave and that the wavelength is long compared to the resonance wavelength of the dye.)

$$
\alpha_{i j}=\alpha_{0}\left[\begin{array}{ll}
1 & 0 \\
0 & 0
\end{array}\right]
$$

If the molecule is inclined toward a principal propagation mode of the host, serving as a Cartesian reference system, we need to rotate $\alpha_{i j}$ by an angle $\sigma$ via a rotation matrix $\mathbf{R}_{\sigma}$ :

$$
\alpha^{\prime}=\mathbf{R}_{\bar{\sigma}}{ }^{T} \alpha \mathbf{R}_{\sigma}=\alpha_{0}\left[\begin{array}{ll}
\cos ^{2} \sigma & \cos \sigma \sin \sigma \\
\cos \sigma \sin \sigma & \sin ^{2} \sigma
\end{array}\right]
$$

Using this matrix $\left(\alpha^{\prime}\right)$, we can calculate $\mathbf{p}$. The resulting scattered dipole waves add to the initial wave at an angle inclined to the initial wave $\mathbf{E}$, thus mimicking OR; the polarization of the electromagnetic wave in the forward direction seems to be tilted about the wave vector.

For an initial polarization along $y$ of the Cartesian reference $\left(\mathbf{E}=\left[0, E_{0}\right]\right)$, the component $p_{x}$ of $\mathbf{p}$ along the $x$ axis of the reference system, normalized to the amplitude of the initial

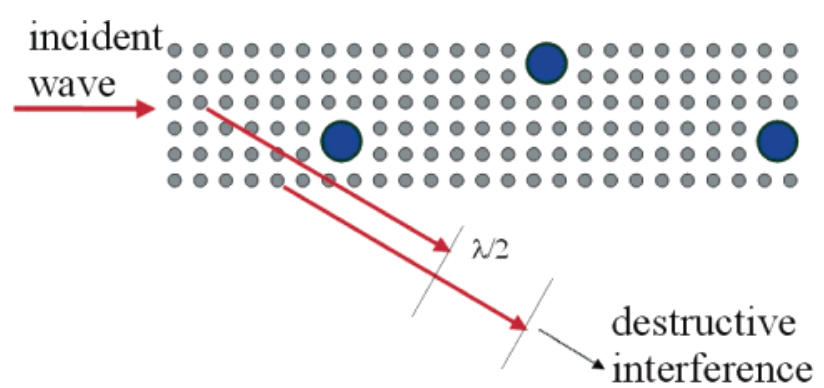

Figure 8. Host molecules (small gray dots) do not contribute to scattering because secondary waves can always be assigned to halfwave retarded pairs. Waves from less dense dye molecules (blue dots) fail to cancel out this way. Waves transmitted from the induced dyedipoles can interfere with the incident light in the forward direction.

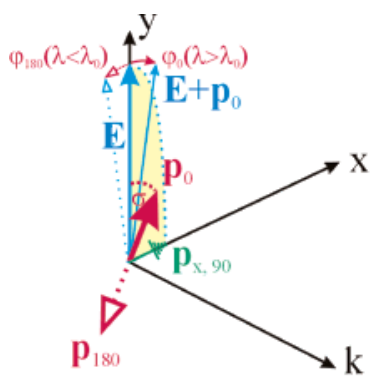

a)

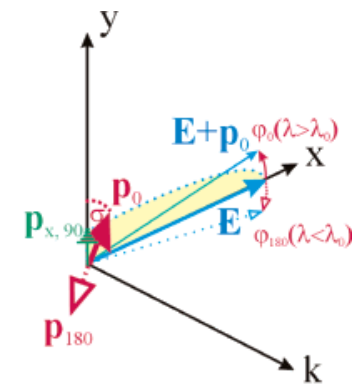

b)
Figure 9. Geometry of optical rotatory and circular dichroic scattering. (a) In the long-wavelength limit $\left(\lambda>\lambda_{0}\right)$, the induced dipole $\mathbf{p}_{0}$ is in phase with the field of the light wave $\mathbf{E}$. The scattered dipole field adds to $\mathbf{E}$, producing dextrorotation of the polarization by an angle $\varphi_{0}$. If the wavelength is short, then $\mathbf{p}$ and $\mathbf{E}$ oscillate with a phase shift of close to $180^{\circ}\left(\mathbf{p}_{180}\right)$. The polarization will be levorotatory $\left(\varphi_{180}\right)$. (b) If the input polarization is chosen parallel to the $x$ axis, then the rotation for long wavelengths is levorotatory, and that for short wavelengths, dextrorotatory. If $\lambda$ is close to the resonance of the dipole, then a phase shift of $90^{\circ}$ occurs that causes the sum of $\mathbf{E}$ and the dipole field transmitted by $\mathbf{p}$ to become elliptically polarized. $\mathbf{p}_{x, 90}$ is the out-ofphase component. $\mathbf{k}$ is the wave vector.

wave, $E_{0}$, is approximately in proportion to the rotation $\varphi_{0}$ of the incoming polarization (Figure 9):

$$
\tan \varphi_{0} \approx \varphi_{0}=p_{x} / E_{0} \approx \alpha_{0} \cos (\sigma) \sin (\sigma), \quad \varphi \text { small }
$$

This term changes sign if the angle $\sigma$ toward the $y$ axis is exchanged with $\sigma+90^{\circ}$. On a macroscopic scale, the sum of all of the dipole waves is transmitted by the induced dipoles along the path of the wave in the crystal. This results in a local electric field that is polarized parallel to the average direction of induced dipoles of the dye molecules, $\langle\mathbf{p}\rangle$, normalized to the incoming field strength. The size of that component and thus 
of the rotation of polarization will depend strongly on the concentration of the molecules in the host crystal.

Thus far we have assumed that the scattered light is far from the dye absorbance band $\left(\lambda_{0}\right)$. This need not be the case. We can distinguish the following three situations: (i) $\lambda>\lambda_{0}$, (ii) $\lambda$ $<\lambda_{0}$, and (iii) $\lambda=\lambda_{0}$. Whereas ORS has different signs in the (i) long- and (ii) short-wavelength (Figure 9) regimes with $\mathbf{E}$ and $\mathbf{p}$ in phase and out of phase (assuming S-shaped dispersion), respectively, CDS is largest when ORS vanishes (iii). Close to resonance, the scattered wave and the initial wave are out of phase by $90^{\circ}$. The superposition of the initial and scattered waves produces an elliptically polarized wave, thus mimicking intrinsic CD. Light linearly polarized along $y$ and passing through a dyed crystal induces the polarization components $i p_{x} x$ and $\mathrm{i} p_{y} y$. The component $\mathrm{i} p_{y} y$ only slightly affects the phaserelation between the large incoming polarization and $\mathrm{i} p_{x} x$. The Jones matrix that will produce these components, $M_{\mathrm{CDS}}$, is given below:

$$
M_{\mathrm{CDS}}=\left[\begin{array}{ll}
1 & \mathrm{i} p_{x} \\
\mathrm{i} p_{x} & 1
\end{array}\right]
$$

Optical properties are often productively comprehended by viewing bulk effects as the sequential consequence of operators for $N$ infinitesimal layers. In our case, $\mathrm{i}\left\langle p_{x}\right\rangle$ is the ellipticity after passing through the whole crystal, and $\delta$ is the phase factor in the Jones matrix for $\mathrm{LB}, M_{\mathrm{LB}}$, given below. The properties of the thin layer are estimated up to the order of $N^{-2}$ from the product of $M_{\mathrm{CDS}}$ and $M_{\mathrm{LB}}$ :

$$
\begin{gathered}
M_{\mathrm{LB}}=\left[\begin{array}{ll}
\mathrm{e}^{\mathrm{i} \delta / 2} & 0 \\
0 & \mathrm{e}^{-\mathrm{i}^{\delta} / 2}
\end{array}\right] \\
M_{\text {layer }}=M_{\mathrm{CDS}} M_{\mathrm{LB}} \approx\left[\begin{array}{ll}
1+\mathrm{i} \delta / 2 N & \mathrm{i}\left\langle p_{x}\right\rangle / N \\
\mathrm{i}\left\langle p_{x}\right\rangle / N & 1-\mathrm{i} \delta / 2 N
\end{array}\right]= \\
\left(1,-\mathrm{i} \frac{\delta}{2 N},-\mathrm{i} \frac{-\left\langle p_{x}\right\rangle}{N}\right)\left(\left[\begin{array}{ll}
1 & 0 \\
0 & 1
\end{array}\right],\left[\begin{array}{ll}
-1 & 0 \\
0 & 1
\end{array}\right],\left[\begin{array}{ll}
0 & 1 \\
1 & 0
\end{array}\right]\right) \\
=\left(1,-\left(\frac{\mathrm{i}}{N} T_{1}\right),-\left(\frac{\mathrm{i}}{N} T_{2}\right)\right)\left(\sigma_{0}, \sigma_{1}, \sigma_{2}\right)=\sigma_{0}-\frac{\mathrm{i}}{N} \mathbf{T} \cdot \sigma
\end{gathered}
$$

According to Schellman and Jensen, ${ }^{19}$ the product matrix can be expressed in terms of the Pauli spin matrices or spinors $\sigma_{0}$, $\sigma_{1}$, and $\sigma_{2}$. The Jones matrix of the whole crystal follows from

$$
M_{\text {crystal }}=\left(\sigma_{0}-\frac{\mathrm{i}}{N} \mathbf{T} \cdot \sigma\right)^{N} \approx \mathrm{e}^{-\mathrm{i} \mathbf{T} \cdot \sigma}
$$

where $\mathbf{T}$ is a mixed circular and linear phase.

$$
\begin{gathered}
\text { With } \mathbf{n}=\frac{\mathbf{T}}{|\mathbf{T}|} \quad|\mathbf{T}| \underset{\left\langle p_{x}\right\rangle \ll \delta}{\longrightarrow} \frac{\delta}{2} \\
(\mathbf{n} \cdot \sigma)^{2 j}=\sigma_{0}, j=1,2,3, \ldots \\
M_{\text {crystal }}=\mathrm{e}^{\mathrm{i}(\delta / 2) \mathbf{n} \cdot \sigma}=\sigma_{0}-\mathrm{i} \frac{\delta}{2} \mathbf{n} \cdot \sigma-\frac{1}{2 !}\left(\frac{\delta}{2} \mathbf{n} \cdot \sigma\right)^{2}+ \\
=\sigma_{0}\left[1-\frac{1}{3 !}\left(\frac{\delta}{2 !} \mathbf{n} \cdot \sigma\right)^{3}+-\ldots\right. \\
\left.\quad \mathrm{in} \cdot \sigma\left[\frac{\delta}{2}-\frac{1}{3 !}\left(\frac{\delta}{2}\right)^{4}+\ldots\right]-\ldots\right]
\end{gathered}
$$

$$
\begin{aligned}
=\sigma_{0} \cos \frac{\delta}{2}- & \mathrm{in} \cdot \sigma \sin \frac{\delta}{2}= \\
& {\left[\begin{array}{ll}
\mathrm{e}^{\mathrm{i}(\delta / 2)} & \mathrm{i}\left(2\left\langle p_{x}\right\rangle / \delta\right) \sin (\delta / 2) \\
\mathrm{i}\left(2\left\langle p_{x}\right\rangle / \delta\right) \sin (\delta / 2) & \mathrm{e}^{-\mathrm{i}(\delta / 2)}
\end{array}\right] }
\end{aligned}
$$

If $i\left\langle p_{x}\right\rangle$ is replaced by an in-phase component $\left\langle p_{x}\right\rangle$, the above treatment produces the Jones matrix for an optically active and linear birefringent crystal. In both cases, we obtain a matrix that is similar to the Jones matrix given in eq 1 of section 2.2.

The off-diagonal matrix elements have the same sign, which is different from that of the elements of the original Jones matrix (eq 1). Nevertheless, after analyzing the HAUP experiment described by eq 2 , we found no difference in the biquadratic polynomial, eq 4. Deviations occur at higher orders in $Y$ and $\Omega$ but are insignificant if $Y$ and $\Omega$ are small $\left(<2^{\circ}\right)$. Thus, CDS and ORS have the same effect in our HAUP measurement as intrinsic OR and CD; however, both new effects depend on the sign of $\left\langle p_{x}\right\rangle$, which changes with a flip of the crystal or a $90^{\circ}$ rotation. (See Figure 9.)

To ensure that LD could not introduce errors into the measurement of $\theta$, we aligned our sample within $0.05^{\circ}$ of $\theta_{0}$ in a clear region. The estimated effect on $\theta$ depends on the transmission ratio, and $\theta_{0}{ }^{\prime}$, the extinction direction in a dyed region. We measured a ratio in the transmission for orthogonal modes of 0.95 in $\mathbf{2}$ and $\sim 0.99$ in $\mathbf{3}$ inside the dyed regions of the thin samples. Thus, the measurement of $\theta$ is affected by LD only approximately ${ }^{28}$ as $(1-0.95) \times 0.05^{\circ}=0.0025^{\circ}$, which is below the resolution of the experiment.

3.2. Comparison with Intrinsic Optical Rotatory Power. ORS and CDS, unlike OR and CD, depend on the incident polarization direction. The effective angle of the superposition of the initial and scattered waves carries different signs for orthogonal incident polarizations or antiparallel wave vectors.

ORS is proportional to the induced electric dipole moment p. It should be much larger than intrinsic OR, which is given as the product of the magnetic and electric dipoles according to Rosenfeld's formulation: $\mathrm{OR} \approx \operatorname{Im}(\mathbf{m} \cdot \mathbf{p}) .{ }^{29}$ As a rule of thumb, the ratio of magnetic and electric dipoles can be equated with the ratio of molecular size to the wavelength of light, about $10^{-3}$ in this case. ${ }^{30}$ Thus, we assume that the contribution to an OR signal due to scattering exceeds the intrinsic molecular contribution by about a factor of $p / m(\sim 1000)$. In the classical view, OR results from induced dipole-dipole exchange. Again, interactions between these dipoles are much smaller than the dipole field that is directly induced by the incident wave in ORS. OR in crystals is typically about $1-1000 \% \mathrm{~mm}$. A $100-\mu \mathrm{m}$ mixed-crystal section where the dye is 1 part in $10^{4}$, given the approximate magnitude of the magnetic dipole, should give an ORS signal of about $0.01-10^{\circ}$. We observed about $1^{\circ}$ (Figures 6 and 7).

The observation of ORS and CDS does not require a chiral scatterer and may be observed with rigid, achiral oscillators so long as they are inclined in the same sense relative to the initial polarization. However, ORS and CDS may be rightfully called chiroptical effects because they are a consequence of mirror symmetry breaking induced by the embedded scatterers similarly inclined with respect to the crystallographic mirror planes. The phenomenological features of ORS and CDS are basically the same as OR and CD, with the exception of the dependence on the polarization of the incident light wave.

3.3. Expectation of Optical Rotatory and Circular Dichroic Scattering. Given the astonishingly thorough studies of the pioneering investigators of light scattering, ${ }^{31}$ we took care 
to be certain that the phenomena described herein had not been observed in previous generations. Lord Rayleigh studied light scattering from defects in chiral crystals. ${ }^{32} \mathrm{He}$ observed that when a pencil of linearly polarized light, traveling along the optic axis of quartz, was scattered at right angles to the direction of propagation alternate maxima and minima were produced as a consequence of the fact that the incident light, rotated successively, produced scattering that was detectable by the observer only when the electric vector was perpendicular to both the direction of propagation and the line of sight of the investigator. In other words, maxima were seen every time the azimuth of the incident light was rotated by $\pi$ or integral multiples thereof. Chandrasekaran, at the urging of Raman, recognized that even pure crystals would show this effect as a consequence of Raman and Brillouin scattering. ${ }^{33 a}$ This, however, is quite distinct from what we see with ORS and CDS in that chirality of the medium is required.

Chandrasekaran further generalized this scattering-fringe phenomenon by showing that achiral, birefringent crystals or strained glasses will also display this periodic scattering for linearly polarized light traveling at some angle with respect to the optic axis. ${ }^{33 a}$ As plane-polarized light advances within a birefringent medium, it undergoes periodic changes in its polarization state. Light scattered transverse to the direction of beam propagation will show maxima once for every integral multiple of $2 \pi$ in the phase factor. Busch and Verreault recognized that the observations of Lord Rayleigh and Chandrasekaran could be used generally to measure the optical rotation and linear birefringence of crystals merely by measuring the spacing between scattering maxima transverse to the direction of light propagation. ${ }^{25}$ Verreault astutely recognized that the azimuth of scattered vibrations from coarse crystals containing scattering centers need not coincide with the polarization of the incident beam. However, this observation was made in the context of a general theory of transverse, scattered radiation in crystals, and his hypothetical example is not matched by experiment. Moreover, ensembles of defects in organized media that preserve the point symmetry of the host will, on average, show the same azimuthal rotation for light scattered and transmitted in the forward direction.

The observation of ORS or CDS requires the coincidence of a number of special circumstances that are met by dyed $\{111\}$ sectors of $\mathrm{K}_{2} \mathrm{SO}_{4}$ :

- The scattering centers must have a strong induced dipole moment.

- The host must not contribute to the rotatory power (OR, ORS, CD, or CDS).

- The angles of the induced dipoles must be inclined to a principal mode of light propagation in the host, and a majority of such molecules must be inclined in the same sense.

- The density of the scatterers must be low enough to allow the observation of scattering and high enough to lift the observed effect over the noise level of polarimetric measurements.

- Finally, state-of-the-art polarimetric analysis is required to detect what nevertheless are small perturbations to the polarization state of the incident light.

Presumably, the unlikely confluence of these conditions explains why these effects, to the best of our knowledge, have not heretofore been reported.

3.4. Outlook. ORS and CDS should be significant in any polarimetric measurements of anisotropic media containing strong oscillators that are oriented with respect to one another and are inclined, though not symmetrically, about the principal vibrational modes. For example, dyes dissolved in a polymer

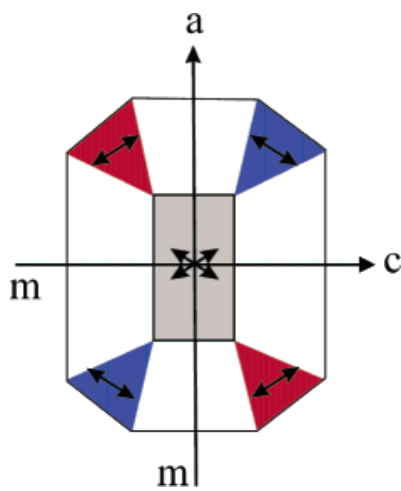

Figure 10. Schematic representation of a plate from a $\mathrm{K}_{2} \mathrm{SO}_{4} / 3$ crystal with $\mathrm{mm}$ symmetry and dyed $\{111\}$ and (010) sectors. The mirror lines are indicated as $\mathrm{m}$. The vectors indicate the absolute orientation of the dipoles, and the colors, the observed sign of ORS (red: positive; blue: negative; gray: no ORS). The three distributions of embedded transition moments have been distinguished from one another by measurements of ORS and CDS. The modulus of the angle was obtained from the LD.

matrix and oriented by uniaxial stretching, further poled by an electric field neither parallel nor perpendicular to the vibration directions fixed by stretching, might serve to produce measurable ORS or CDS. Despite the fact that the orienting of chromophores in stretched polyethylene is a well-developed method for studying the photophysics of solutes, ${ }^{30}$ we are unaware of any occasion whereby it would have been profitable to "tip" oriented chromophores similarly clockwise (or counterclockwise) with respect to the stretching direction. Moreover, stretched polymers tend to have strong LB. Since ORS and CDS vary as $\sin (\delta) / \delta$, scattering would be hard to observe even if such a material had been confronted in the past with a polarimeter.

ORS and CDS might be significant in dyed, chiral tissues. Histology is rife with stained biological structures. If the structures are anisotropic, then the dyes will form dilute oriented glasses. If the structures are also chiral, there is no expectation that the dyes would be oriented with the vibrational directions of the medium. However, in chiral media, intrinsic OR and CD could be dominant - these are absent in centrosymmetric $\mathrm{K}_{2} \mathrm{SO}_{4}-$ thus obviating the possibility of observing the new effects. Biogenic crystals contain dilute oriented glasses of chiral proteins. Whereas most biominerals are achiral (e.g., $\mathrm{CaCO}_{3}$ ), the dipole moments in chiral chromophores embedded in achiral crystals will not necessarily have their dipoles oriented with the principal modes of the medium.

Measurements of OR have been plagued in the past by socalled "parasitic ellipticities" believed to be related to the optical components of the experimental devices with which the measurements were performed. One strategy for minimizing parasitic ellipticities has been to average measurements differing by $90^{\circ}$ rotations of the sample. ${ }^{18}$ We see here that scattering from defects leading to ORS and CDS could have contributed to the parasitic effects.

ORS and CDS can also be used as unique probes of structure. In the past, we have studied dyed crystals by making measurements of LD. ${ }^{8}$ However, such measurements can be limited by the symmetry of the host crystal. For example, for incident light normal to an orthorhombic $\mathrm{K}_{2} \mathrm{SO}_{4}$ plate, we cannot know whether the transition moments for the included dyes are inclined to the left or to the right or in both directions (Figure 10 , (010) growth sector). This limitation is a consequence of the fact that in an orthorhombic crystal the propagation modes for light are parallel to the crystallographic axes. The electric field will sample the crystal in the same way irrespective of 
the direction of the inclination of the incident polarization with respect to the vibrational directions; the components of the incident radiation that are separated as the ordinary and extraordinary rays will be identical in either case. This limitation is a manifestation of the Neumann-Curie principle; ${ }^{34}$ we cannot subvert the symmetry of the crystal. However, scattering is a consequence of light interactions with isolated molecules that do not manifest the symmetry of the crystal. Thus, we can now determine absolutely the directionality of the oscillator inclination, and we have devised a new structural probe of the materials that we set out to examine.

Acknowledgment. We are grateful for support from the National Science Foundation, the donors of the American Chemical Society Petroleum Research Fund, the University of Washington Center for Nanotechnology, and the FPU research grant from the Ministerio de Educación y Cultura (Spain). We thank Dr. S.-H. Jang for synthesizing brilliant Congo R and L. D. Bastin for assistance in growing crystals.

\section{References and Notes}

(1) Kaminsky, W. Rep. Prog. Phys. 2000, 63, 1575-1640.

(2) Goldschmidt, V. M. New Biol. 1952, 12, 97-105.

(3) Bernal, J. D. The Physical Basis of Life; Routledge and Paul: London, 1951

(4) Cairns-Smith, A. G. Genetic Takeover and the Mineral Origins of Life; Cambridge University Press: Cambridge, U.K., 1987.

(5) (a) Addadi, L.; Berkovitch-Yellin, Z.; Weissbuch, I.; Lahav, M.; Leiserowitz, L. J. Am. Chem. Soc. 1982, 104, 2075-2077. (b) Addadi, L.; Berkovitch-Yellin, Z.; Weissbuch, I.; Mil, J. v.; Shimon, L. J. W.; Lahav, M.; Leiserowitz, L. Angew. Chem., Int. Ed. Engl. 1985, 24, 466-485. (c) Addadi, L.; Berkovitch-Yellin, Z.; Weissbuch, I.; Lahav, M.; Leiserowitz, L. In Topics in Stereochemistry; Eliel, E. L., Willen, S. H., Allinger, N. L., Eds.; John Wiley and Sons: New York, 1991; pp 1-85. (d) Vaida, M.; Shimon, L. J. W.; Weisinger-Lewin, Y.; Frolow, F.; Lahav, L.; Leiserowitz, L.; McMullan, R. K. Science (Washington, D.C.) 1988, 241, 1475-1479. (e) Weissbuch, I.; Popovitz-Biro, R.; Lahav, M.; Leiserowitz, L. Acta Crystallogr., Sect. B 1995, 51, 115-148.

(6) (a) Bondy, S. C.; Harrington, M. E. Science (Washington, D.C.) 1979, 23, 1243-1244. (b) Cody, A. M.; Cody, R. D. J. Cryst. Growth 1991, $113,508-519$.

(7) (a) Bonner, W. A.; Kavasmaneck, P. R.; Martin, F. S.; Flores, J. J. Origins Life 1975, 6, 367-376. (b) Kavasmaneck, P. R.; Bonner, W. A. J. Am. Chem. Soc. 1977, 99, 44-50.

(8) Kahr, B.; Gurney, R. W. Chem. Rev. 2001, 101, 893-951.

(9) (a) Buckley, H. E. Z. Kristallogr. 1934, 88, 248-255. See also (b) Buckley, H. E. Crystal Growth; John Wiley: New York, 1951.

(10) Colour Index, 1st ed. Rowe, F. M., Ed.; Society of Dyers and Colourists: Bradford, Yorkshire, U.K., 1924. Colour Index, 2nd ed. Society of Dyers and Colourists: Bradford, Yorkshire, U.K., 1956. Colour Index, 3rd ed. Society of Dyers and Colourists: Bradford, Yorkshire, U.K., 19711982.

(11) At room-temperature $\mathrm{K}_{2} \mathrm{SO}_{4}$ crystallizes in the orthorhombic space group Pnma with $a=7.483 \AA, b=5.772 \AA, c=10.072 \AA$. ((a) Wyckoff,
R. W. G. Crystal Structures, v.3. Wiley-Interscience: New York, 1965; Vol. 3, pp 95-97). (b) Ojima, K.; Nishihata, Y.; Sawada, A. Acta Crystallogr. Sect. B, 1995, B51, 287-293.) To be consistent with Buckley, our Miller indices refer to ratios determined by classical goniometry where $b>c>a$ in the nonstandard setting Pcnm. (c) von Groth, P. Chemische Krystallographie; W. Engelmann: Leipzig, Germany, 1908; Vol. 2, p 351. (d) Tutton, A. E. J. Chem. Soc. 1894, 628-717.

(12) Zollinger, H. Color Chemistry, 2nd ed. VCH: Weinheim, Germany, 1991.

(13) Chow, J. K.; Kelley, M. P.; Kahr, B. Mol. Cryst. Liq. Cryst. 1994, $242,201-214$.

(14) This is distinct from circular intensity differential scattering. (a) Bustamante, C.; Maestre, M. F.; Tinoco, I., Jr. J. Chem. Phys. 1980, 73, 4273, 6046. (b) Bustamante, C.; Tinoco, I., Jr.; Maestre, M. F. J. Chem. Phys. 1981, 74, 4839. (c) Bustamante, C.; Tinoco, I., Jr.; Maestre, M. F. J. Chem. Phys. 1982, 76, 3440. (d) Bustamante, C. Circular Intensity Differential Scattering. Ph.D. Dissertation, University of California, Berkeley, CA, 1980.

(15) Bastin, L.; Kahr, B. Tetrahedron 2000, 56, 6633-6643.

(16) Wedding, W. Ann. Phys. 1888, 35, 25. Wiener, O. Ann. Phys. 1888 $35,1-24$.

(17) Szivessy, G.; Münster, C. Ann. Phys. 1934, 20, 703-736.

(18) (a) Kobayashi, J.; Uesu, Y. J. Appl. Crystallogr. 1983, 16, 204211. (b) Kobayashi, J.; Uesu, Y.; Takahashi, H. J. Appl. Crystallogr. 1983, 16, 212-219.

(19) Schellman, J.; Jensen, H. P. Chem. Rev. 1987, 87, 1359-1399.

(20) Moxon, J. R. L.; Renshaw, A. R. J. Phys.: Condens. Matter 1990, 2, 6807-6836.

(21) Winchell, A. N.; Winchell, H. The Microscopical Charcters of Artifical Inorganic Solid Substances: Optical Properties of Artificial Minerals; McCrone Research Institute: Chicago, IL, 1989; p 125.

(22) Kaminsky, W.; Glazer, A. M. Ferroelectrics 1996, 183, 133-141.

(23) (a) Kaminsky, W. Phase Transitions 1996, 59, 121-133. (b) Kaminsky, W. Ferroelectrics 1997, 204, 233-246. (c) Kaminsky, W.; Glazer, A. M. Phase Transitions 1998, 66, 1-21.

(24) van der Hulst, H.-C. Light Scattering by Small Particles; Dover Publications: Mineola, NY, 1981.

(25) Busch, G.; Verreault, R. Acta Crystallogr., Sect. A 1971, 27, 680681. Verrault, R. Z. Kristallogr. 1972, 136, 350-386.

(26) In the long-wavelength limit, only lower-order multipoles will be significant.

(27) Sowronek, M.; Roterman, I.; Konieczny, L.; Stopa, B.; Rybarska, J.; Piekarska, B.; Górecki, A.; Król, M. Comput. Chem. (Oxford) 2000, 24, 429-450. Sowronek, M.; Roterman, I.; Konieczny, L.; Stopa, B.; Rybarska, J.; Piekarska, B. J. Comput. Chem. 2000, 21, 656-667.

(28) Kaminsky, W.; Haussühl, S. Z. Kristallogr. 1993, 203, 79-91.

(29) Rosenfeld, L. Z. Phys. 1928, 52, 161-174.

(30) Michl, J.; Thulstrup, E. W. Spectroscopy with Polarized Light: Solute Alignment by Photoselection, in Liquid Crystals, Polymers, and Membranes, VCH: Deerfield Beach, FL, 1995; p 33.

(31) Strutt, R. J. Scientific Papers; Dover Publications: Mineola, NY, 1964. Raman, C. V. Scattering of Light. In Scientific Papers; Ramaseshan, S., Ed.; Indian Academy of Sciences: Bangalore, India, 1988; Vol. I.

(32) Strutt, R. J. Proc. R. Soc., London, Ser. A 1919, 95, 476-470.

(33) (a) Chandrasekharan, V. Proc. Indian Acad. Sci., Sect. A 1947, 25 , 256-259. (b) Chandrasekharan, V. Proc. Indian Acad. Sci., Sect. A 1948, A28, 409-415.

(34) Kaminsky, W.; Kahr, B. Symmetry 2000; Wenner-Gren Foundation: Stockholm, 2001; pp 309-316. 